\title{
Brief Communication: Operative Fixation for Distal Radius Fracture Increases Rates of Trigger Finger Over a 6-Month Post-Operative Period
}

\author{
Lauren E Wessel*, Alex Gu, Francesca Coxe, Jeffrey G Stepan, Duretti T Fufa and \\ Daniel A Osei \\ Hospital for Special Surgery, USA
}

\section{ISSN: 2576-8875}

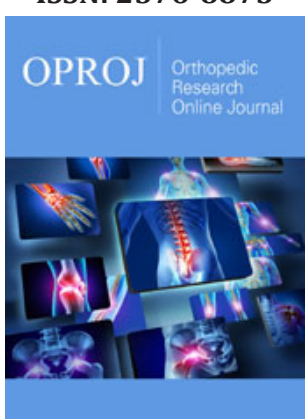

*Corresponding author: Lauren E Wessel, Hospital for Special Surgery, USA

Submission: 㽬 January 23, 2020

Published: 眥February 04, 2020

Volume 6 - Issue 4

How to cite this article: Lauren E Wessel, Alex Gu, Francesca Coxe, Jeffrey G Stepan, Duretti T Fufa, et al. Brief Communication: Operative Fixation for Distal Radius Fracture Increases Rates of Trigger Finger Over a 6-Month Post-Operative Period. Ortho Res Online J. 6(4). OPROJ.000642.2020. DOI: 10.31031/OPROJ.2020.06.000642

Copyright@: Lauren E Wessel, This article is distributed under the terms of the Creative Commons Attribution 4.0 International License, which permits unrestricted use and redistribution provided that the original author and source are credited.

\begin{abstract}
The primary purpose of this study was to elucidate the relationship between trigger finger (TF) and open reduction and internal fixation (ORIF) for distal radius fracture (DRF), comparing the incidence of TF in patients who underwent DRF ORIF to the incidence of TF in patients who underwent nonoperative DRF treatment. Data were collected from the Humana Insurance subset database of the pearl diver system for all patients who underwent ORIF for DRF between 2015 and 2017. Incidence of trigger finger was determined for extremities based upon the presence of a trigger finger diagnostic International classification of diseases (ICD-10) code within 6 months of ORIF. In our cohort of 23,733 patients, distal radius ORIF was an independent risk factor for trigger finger development subsequent to DRF (OR: 1.33, 95\% CI: $1.23-1.43 ; \mathrm{p}<0.001)$. Surgeons should be aware of this association, particularly during post-operative patient assessment.
\end{abstract}

\section{Introduction}

Fractures of the distal radius are common, accounting for almost $20 \%$ of fractures in patients over the age of 65 [1]. While management of these fractures is highly dependent on a variety of fracture- and patient-specific factors, rates of open reduction internal fixation (ORIF) for distal radius fracture (DRF) have more than doubled over the past 10 years [2,3]. The reported complication rate following DRF varies from 6\% to 80\%, with a recent study by McKay et al. [4] citing a complication rate of $17 \%$ in patients who underwent DRF ORIF $[4,5]$. Nerve injury complications following DRF, particularly involving the median nerve, are relatively common and regularly reported, with a variable incidence of $0 \%$ to $17 \%$ [5]. On the other hand, tendon complications, including rupture, adhesions, tenosynovitis, and trigger finger (TF), are rarer events. While the specific etiology of TF post-DRF has not been elucidated, trigger finger reportedly occurs in $2 \%$ to $6.5 \%$ of cases across all treatment modalities $[5,6]$.

The relationship between TF and carpal tunnel syndrome (CTS) is well known, with the two pathologies occurring concomitantly in many cases $[7,8]$. TF occurs at higher rates in extremities affected by CTS, rheumatoid arthritis and hypothyroidism, supporting the belief that TF is likely associated with the existence of an inflammatory process [8]. Moreover, a recent retrospective study by Blazar et al found that new onset TF is 2.5-times more likely to develop in a hand 6 months post-carpal tunnel release as compared to the contralateral nonoperative hand [8]. Given the co-occurrence of TF and both systemic inflammatory processes as well as post-surgical states, we hypothesized that ORIF for DRF may be an independent risk factor for the development of TF.

The primary purpose of this study was to elucidate the relationship between TF and DRF ORIF, comparing the incidence of TF in patients who underwent DRF ORIF to the incidence of $\mathrm{TF}$ in patients who underwent nonoperative DRF treatment. Our hypothesis was that extremities/patients undergoing DRF ORIF are at increased risk of developing trigger finger during a 6-month postoperative period compared to extremities managed conservatively. A secondary aim was to see whether there were any predictive factors for development of TF after DRF utilizing multivariate analysis. Finally, we compared rates of TF development in the ORIF group to the contralateral extremity in order to compare the rate to that of the natural history of TF development in this population. 


\section{Materials and Methods}

Data were collected from the Humana Insurance subset database of the PearlDiver System for all patients who underwent ORIF for DRF between 2015 and 2017. Demographic information and medical comorbidities were recorded. Selection of comorbidity variables was based upon prior work conducted by Elixhauser et al. [9] Patients with bilateral DRFs, prior history of trigger finger, distal radius fractures, less than 6 months of follow up, or unknown laterality were excluded.

Patients were identified based upon ICD-10 code for distal radius fracture (Appendix 1) and stratified by operative versus non-operative management of DRF. Incidence of trigger finger was determined for extremities based upon the presence of a trigger finger diagnostic International Classification of Diseases (ICD-10) code within 6 months of ORIF. ICD-10 coding allowed for control of laterality. The contralateral extremity in our cohort of patients who underwent ORIF of DRF was used as an internal control group. Patients with bilateral DRFs, prior history of trigger finger, distal radius fractures, less than 6 months of follow up, or unknown laterality were excluded.

We performed binary logistic regression to model risk factors for the development of TF after DRF utilizing the entire population of patients who sustained DRF during the relevant timeframe. Rates of trigger finger among the cohorts were analyzed using Pearson's Chi-square on R software provided within PearlDiver. The results were reported as odds-ratios and 95\% confidence intervals. A p-value of $<0.05$ was used as the cutoff for significance.

\section{Appendix 1}

Table 1: CPT and ICD codes.

\begin{tabular}{|c|r|}
\hline Category & \multicolumn{1}{|c|}{ Codes } \\
\hline \multirow{5}{*}{$\begin{array}{c}\text { ICD-10-D-S52501, ICD-10-D-S52501A, ICD-10-D-S52501B, ICD-10-D-S52501C, ICD-10-D-S52511, ICD-10-D-S52511A, } \\
\text { ICD-10-D-S52511B, ICD-10-D-S52511C, ICD-10-D-S52514, ICD-10-D-S52514A, ICD-10-D-S52514B, ICD-10-D-S52514C, } \\
\text { ICD-10-D-S52521, ICD-10-D-S52521A, ICD-10-D-S52531, ICD-10-D-S52531A, ICD-10-D-S52531B, ICD-10-D-S52531C, } \\
\text { ICD-10-D-S52541, ICD-10-D-S52541A, ICD-10-D-S52541B, ICD-10-D-S52541C, ICD-10-D-S52551, ICD-10-D-S52551A, } \\
\text { ICD-10-D-S52551B, ICD-10-D-S52551C, ICD-10-D-S52561, ICD-10-D-S52561A, ICD-10-D-S52561B, ICD-10-D-S52561C, } \\
\text { ICD-10-D-S52571, ICD-10-D-S52571A, ICD-10-D-S52571B, ICD-10-D-S52571C, ICD-10-D-S52591, ICD-10-D-S52591A, } \\
\text { ICD-10-D-S52591B, ICD-10-D-S52591C, ICD-10-D-S52502, ICD-10-D-S52502A, ICD-10-D-S52502B, ICD-10-D-S52502C, } \\
\text { ICD-10-D-S52512, ICD-10-D-S52512A, ICD-10-D-S52512B, ICD-10-D-S52512C, ICD-10-D-S52515, ICD-10-D-S52515A, } \\
\text { ICD-10-D-S52515B, ICD-10-D-S52515C, ICD-10-D-S52522, ICD-10-D-S52522A, ICD-10-D-S52532, ICD-10-D-S52532A, } \\
\text { ICD-10-D-S52532B, ICD-10-D-S52532C, ICD-10-D-S52542, ICD-10-D-S52542A, ICD-10-D-S52542B, ICD-10-D-S52542C, } \\
\text { ICD-10-D-S52552, ICD-10-D-S52552A, ICD-10-D-S52552B, ICD-10-D-S52552C, ICD-10-D-S52562, ICD-10-D-S52562A, } \\
\text { ICD-10-D-S52562B, ICD-10-D-S52562C, ICD-10-D-S52572, ICD-10-D-S52572A, ICD-10-D-S52572B, ICD-10-D-S52572C, } \\
\text { ICD-10-D-S52592, ICD-10-D-S52592A, ICD-10-D-S52592B, ICD-10-D-S52592C }\end{array}$} \\
\hline $\begin{array}{r}\text { Open Reduction Internal } \\
\text { Fixation }\end{array}$ & $\begin{array}{r}\text { CPT-25515, CPT-25525, CPT-25526, CPT-25545, CPT-25574, CPT-25575, CPT-25607, CPT-25608, CPT-25609, CPT- } \\
\text { 25651, CPT-25652, CPT-25515, CPT-25525 }\end{array}$ \\
\hline \multirow{2}{*}{$\begin{array}{r}\text { Trigger Finger } \\
\text { ICD-10-D-M65311, ICD-10-D-M65321, ICD-10-D-M65331, ICD-10-D-M65341, ICD-10-D-M65351, ICD-10-D-M65312, } \\
\text { ICD-10-D-M65322, ICD-10-D-M65332, ICD-10-D-M65342, ICD-10-D-M65352 }\end{array}$} \\
\hline
\end{tabular}

\section{Results}

In total, 23,733 patients were diagnosed with DRF (average age: 71 years, range: $20-96 ; 74.6 \%$ female). Among those patients, 4,967 patients (20.9\%) underwent ORIF and 18,766 (79.1\%) did not. Among the operative and non-operative cohorts, 61 patients $(1.2 \%)$ and 108 patients $(0.5 \%)$ received treatment for trigger finger, respectively. Demographics for groups are listed in Table 1 and demonstrated significant differences between cohorts managed with ORIF versus conservatively.

Given the existence of significant differences between cohorts, multivariate analysis was performed to determine independent risk factors for the development of TF. Following multivariate analysis, patients treated with ORIF had an increased odds risk of 1.33 for trigger finger development following distal radius fracture $(95 \%$ CI: 1.23-1.43; p<0.001). Diabetes (OR: 1.27; 95\% CI: 1.19-1.35; $\mathrm{p}<0.001$ ), older age (OR: 3.41; 95\% CI: 2.96-3.878; p<0.001), and depression (OR: 1.26: 95\% CI: 1.16-1.36; p<0.001) were also found to be significant predictors for the development of trigger finger after distal radius fracture.

In total, 4,967 patients underwent ORIF within the relevant time period. Baseline patient demographics and clinical characteristics are further listed in Table 1. In total, 61 extremities (1.2\%) in the operative cohort and 31 patients $(0.6 \%)$ in the internal control cohort developed postoperative trigger finger. Compared to the internal control cohort, extremities that underwent ORIF for DRF had an increased rate of developing trigger finger in the immediate 6 -month postoperative period $(1.2 \%$ vs $0.6 \%, p=0.0052)$. The average pooled time from ORIF to trigger finger diagnosis was 4.4 months (SD: 1.1 months; range: 0.1-6.0 months), with no significant difference observed between the two cohorts. There was no significant difference in the distribution of fingers affected by triggering between the two cohorts. 
Table 1: Demographics of ORIF and conservative DRF management groups .

\begin{tabular}{|c|c|c|c|c|c|}
\hline & \multicolumn{2}{|c|}{ ORIF } & \multicolumn{2}{|c|}{ Conservative } & \multirow[t]{3}{*}{ p-value } \\
\hline & \multicolumn{2}{|c|}{4967} & \multicolumn{2}{|c|}{18766} & \\
\hline & $\mathbf{n}$ & $\%$ & $\mathbf{n}$ & $\%$ & \\
\hline Gender & & & & & $<0.001$ \\
\hline Male & 828 & $16.67 \%$ & 5187 & $27.64 \%$ & \\
\hline Female & 4139 & $83.33 \%$ & 13579 & $72.36 \%$ & \\
\hline Age & & & & & $<0.001$ \\
\hline Under 50yr & 434 & $8.74 \%$ & 4342 & $23.14 \%$ & \\
\hline $50-59 y r$ & 592 & $11.92 \%$ & 1450 & $7.73 \%$ & \\
\hline $60-69 y \mathrm{r}$ & 1425 & $28.69 \%$ & 3486 & $18.58 \%$ & \\
\hline 70-79yr & 1764 & $35.51 \%$ & 5018 & $26.74 \%$ & \\
\hline $80-89 y \mathrm{yr}$ & 678 & $13.65 \%$ & 3437 & $18.32 \%$ & \\
\hline Over 90yr & 74 & $1.49 \%$ & 1033 & $5.50 \%$ & \\
\hline Diabetes Mellitus & 781 & $15.72 \%$ & 1728 & $9.21 \%$ & $<0.001$ \\
\hline Hypothyroidism & 591 & $11.90 \%$ & 837 & $4.46 \%$ & $<0.001$ \\
\hline Rheumatoid or Collagen Disease & 73 & $1.47 \%$ & 206 & $1.10 \%$ & $<0.001$ \\
\hline Depression & 487 & $9.80 \%$ & 680 & $3.62 \%$ & $<0.001$ \\
\hline Fracture Severity (Type 3 A, B, C) & 18 & $0.36 \%$ & 34 & $0.18 \%$ & 0.024 \\
\hline Trigger Finger & 45 & $0.91 \%$ & 108 & $0.58 \%$ & $<0.001$ \\
\hline
\end{tabular}

\section{Discussion and Conclusion}

In our cohort of 23,733 patients, distal radius ORIF was an independent risk factor for trigger finger development subsequent to DRF. These data are in line with recent literature demonstrating that patient's status-post carpal tunnel release demonstrate high rates of TF than those treated conservatively [8]. Additionally, these data are supported by the widely ascribed to belief that TF is caused in part by an inflammatory state, and our study further confirmed risk factors for the development of TF, including diabetes, age and depression [10,11].

Our brief study had inherent limitations. Similar to all studies conducted on administrative data, we were unable to characterize post-treatment outcomes with regard to either satisfaction or functional parameters. For similar reasons, we were unable to determine whether the increased rate of $\mathrm{TF}$ development was observed in the operative DRF group was secondary to surgery itself or to fracture severity. Further prospective studies with appropriate propensity matching according to fracture severity and mechanism of trauma would be required to further address these questions. Finally, our follow-up period was limited to 6-months. However, this was set intentionally so as to capture the incidence of TF associated with acute DRF rather than the incidence associated with the natural history of TF in this population.

Our study clarified the relationship between TF and DRF ORIF, comparing the incidence of TF in patients who underwent DRF ORIF to the incidence of TF in patients who underwent nonoperative DRF treatment. Our study demonstrated an increased risk of TF with operative management of distal radius fracture. Although we do not suggest change in management for distal radius fracture based on our data, we do believe that knowledge of these data is critical to both post-operative examination and surveillance as well as pre-operative patient counseling. Surgeons should be aware of this association, particularly during post-operative patient assessment.

\section{References}

1. Baron JA, Karagas M, Barrett J (1996) Basic epidemiology of fractures of the upper and lower limb among Americans over 65 years of age. Epidemiology 7(6): 612-618.

2. Nellans KW, Kowalski E, Chung KC (2012) The epidemiology of distal radius fractures. Hand Clin 28(2): 113-125.

3. Levin LS, Rozell JC, Pulos N (2017) Distal radius fractures in the elderly. J Am Acad Orthop Surg 25(3): 179-187.

4. McKay SD, MacDermid JC, Roth JH, Richards RS (2001) Assessment of complications of distal radius fractures and development of a complication checklist. J Hand Surg Am 26(5): 916-922.

5. Turner RG, Faber KJ, Athwal GS (2010) Complications of distal radius fractures. Hand Clin 26(1): 85-96.

6. Chung KC, Malay S, Shauver MJ, Kim HM (2019) Assessment of distal radius fracture complications among adults 60 years or older. JAMA Netw Open 2(1): e187053.

7. Kim JH, Gong HS, Lee HJ, Lee YH, Rhee SH, et al. (2013) Pre- and postoperative comorbidities in idiopathic carpal tunnel syndrome: Cervical arthritis, basal joint arthritis of the thumb, and trigger digit. J Hand Surg Eur 38(1): 50-56. 
8. Zhang D, Collins J, Earp BE, Blazar P (2019) Relationship of carpal tunnel release and new onset trigger finger. J Hand Surg Am 44(1): 28-34.

9. Elixhauser A, Steiner C, Harris D, Coffey R (1998)Comorbidity measures for use with administrative data. Med Care 36(1): 8-27.

10. Makkouk AH, Oetgen ME, Swigart CR, Dodds SD (2008) Trigger finger: etiology, evaluation, and treatment. Curr Rev Musculoskelet Med 1(2): 92-96.

11. Wessel LE, Fufa DT, Boyer MI, Calfee RP(2013) Epidemiology of carpal tunnel syndrome in patients with single versus multiple trigger digits. J Hand Surg Am 38(1): 49-55.

For possible submissions Click below: 